Ian D. Wilson · Gary L. A. Barker · Chungui Lu •

Jane A. Coghill $\cdot$ Richard W. Beswick •

John R. Lenton · Keith J. Edwards

\title{
Alteration of the embryo transcriptome of hexaploid winter wheat (Triticum aestivum cv. Mercia) during maturation and germination
}

Received: 5 November 2004 / Revised: 11 January 2005 / Accepted: 12 January 2005 / Published online: 16 February 2005 (C) Springer-Verlag 2005

\begin{abstract}
Grain dormancy and germination are areas of biology that are of considerable interest to the cereal community. We have used a 9,155-feature wheat unigene cDNA microarray resource to investigate changes in the wheat embryo transcriptome during late grain development and maturation and during the first $48 \mathrm{~h}$ of postimbibition germination. In the embryo 392 mRNAs accumulated by twofold or greater over the time course from 21 days postanthesis (dpa) to $40 \mathrm{dpa}$ and on through 1 and 2 days postgermination. These included mRNAs encoding proteins involved in amino acid biosynthesis and metabolism, cell division and subsequent cell development, signal transduction, lipid metabolism, energy production, protein turnover, respiration, initiation of transcription, initiation of translation and ribosomal composition. A number of mRNAs encoding proteins of unknown function also accumulated over the time course. Conversely 163 sequences showed decreases of twofold or greater over the time course. A small number of mRNAs also showed rapid accumulation
\end{abstract}

The EMBL accession numbers for sequence data for the sequences used in the construction of the microarrays described in this report are AL808216-AL831324.

Electronic Supplementary Material Supplementary material is available for this article at http://dx.doi.org/10.1007/s10142-0050137-2.

I. D. Wilson $(\bowtie) \cdot$ G. L. A. Barker · C. Lu · J. A. Coghill ·

R. W. Beswick - J. R. Lenton - K. J. Edwards

Department of Biological Sciences, University of Bristol,

Woodland Road,

Bristol, BS8 1UG, UK

e-mail: ian.wilson@bristol.ac.uk

Tel.: +44-117-3317085

Fax: +44-117-9257374

C. $\mathrm{Lu}$

Rothamsted Research,

Harpenden, Hertfordshire, AL5 2JQ, UK

R. W. Beswick

Department of Haematology, Hammersmith Hospital,

Du Cane Road,

London, W12 0NN, UK specifically during the first $48 \mathrm{~h}$ of germination. We also examined alterations in the accumulation of transcripts encoding proteins involved in abscisic acid signalling. Thus, we describe changes in the level of transcripts encoding wheat Viviparous 1 (Vp1) and other interacting proteins. Interestingly, the transcript encoding wheat Viviparous-interacting protein 1 showed a pattern of accumulation that correlates inversely with germination. Our data suggests that the majority of the transcripts required for germination accumulate in the embryo prior to germination and we discuss the implications of these findings with regard to manipulation of germination in wheat.

Keywords Triticum aestivum - Microarray · Dormancy · Germination · Embryo

\section{Introduction}

Seed dormancy, defined as the failure of the intact seed to complete germination under favourable conditions (Bewley 1997), and germination are complex adaptive traits of higher plants that are influenced by a large number of genes and environmental factors (Koornneef et al. 2002). The ability of a seed to germinate has been described as a balance between the growth potential of the embryo under promoting conditions and the restrictions imposed by the surrounding tissues. In wheat this balance is a particularly important consideration, which is strongly influenced by the genotype and its interaction with the environment during grain development. The potential for early germination of the seed within the ear, known as preharvest sprouting (PHS), has significant implications with regard to the breadmaking quality of the particular wheat variety in question. A rise in the level of hydrolytic enzymes in the maturing grain occurring during periods of cool, damp weather just prior to PHS can result in considerable loss of grain quality (McKibbin et al. 2002).

Quantitative trait loci (QTL) affecting seed dormancy and germination have been reported for wheat (Kato et al. 2001; Osa et al. 2003). In dicotyledonous plants, where the 
embryonic axis of the germinating seed has to digest the surrounding endosperm prior to emergence, mutations in a number of genes are known to have direct, indirect and/or additive effects on seed dormancy and germination and the use of mutants such as the abscisic acid (ABA) insensitive (abi; Koornneef et al. 1984) and enhanced response to ABA (era) series (for review see Finkelstein and Zeevaart 1994) has identified the role of ABA in seed dormancy as well as the requirement of gibberellic acid (GA) for germination (Debeaujon and Koornneef 2000; Agrawal et al. 2001). In cereals, where the embryo of the germinating seed simply emerges through the pericarp, the situation is less clear. However, physiological experiments involving different wheat varieties indicate that wheat germination is similarly inhibited by ABA with a sensitivity dependent on the genotype (Himi et al. 2002). Mutation of the Viviparousl gene (Vpl), a DNA-binding (Suzuki et al. 1997) acidic transcriptional activator (McCarty et al. 1991) first identified in maize and orthologous to the Arabidopsis thaliana ABI3 gene (Giraudat et al. 1992), causes reduced expression of some enzymes and seed maturation-related genes (Hattori et al. 1994) while promoting germination of embryos still attached to the cob. Severe mutations of the $A B I 3$ gene lead to premature activation of the apical meristem during embryo maturation, a lack of accumulation of seed storage products and activation of the promoter of the germination-related $C A B 3$ gene before seed shed (Nambara et al. 1995). In addition to $A$. thaliana, orthologues of $V p 1$ have been cloned for a number of species including rice (Hattori et al. 1994) and wild oat (Jones et al. 1997) and in wheat homeologues have been mapped to the long arms of the group-3 chromosomes at a location conserved relative to $V p 1$ in maize and to the orthologue in rice (Bailey et al. 1999). In modern wheat and in the ancestral species $V p 1$ transcripts appear to be misspliced and it has been suggested that this may contribute to the susceptibility of some modern hexaploid wheat varieties to PHS (McKibbin et al. 2002). Proteins that interact with the gene products of the wild oat $V p 1$ orthologue and the $A B I 3$ gene have also been identified that show differential expression in the dormant and germinating embryo and may play specific roles in regulating the transition from dormancy to germination (Jones et al. 2000). A rice bZIP factor that interacts with $\mathrm{Vp} 1$ and may mediate ABA signalling via interaction with ABA responsive elements has also been cloned (Hobo et al. 1999). Recent gene chip array experiments in Arabidopsis also suggest that $A B I 3$ exerts its effect on germination by regulating ABA signalling (Suzuki et al. 2003). In addition to these specific genes that have been identified by genetic approaches there are also a number of genes that have been identified as important in that the accumulation patterns of their respective mRNAs correlate temporally and spatially with embryo maturation, dormancy and germination. A number of microarray experiments with $A$. thaliana seeds have also confirmed prior knowledge and simultaneously identified a number of novel genes and proteins associated with dormancy and germination (Girke et al. 2000; Gallardo et al. 2001, 2002a). For wheat far less is understood concerning the mechanisms underlying and the genes involved in seed dormancy and germination. Here we describe a microarray-based investigation that provides new insights into the wheat genes with expression patterns correlated with these developmental processes. We also describe the use of a publicly available wheat transcriptomics resource and provide considerable new information about the gene expression underlying the biology of germination in the cereals.

\section{Materials and Methods}

Plant material, RNA extractions, microarray production and hybridisation

Embryos were isolated from developing wheat grains at 21 and 40 days postanthesis (dpa) and at 1 and 2 days postgermination (dpg; postimbibition) as described in Wilson et al. (2004). Total RNA samples were extracted from the tissues using a modification of the approach described by Chen et al. (2001) and outlined in Wilson et al. (2004).

The production of cDNA microarrays of a wheat unigene set on Codelink activated slides (Amersham Biosciences, Chalfont St. Giles, UK), the generation of Alexa Fluor 555 and 647 dye- (Molecular Probes, Eugene, Ore.) labelled hybridisation extracts and their hybridisation to the arrays in dye swap experiments was as described by Wilson et al. (2004). The resulting hybridisation signals were visualised by scanning using an Axon Instruments GenePix 4000B dual laser microarray scanner (Axon Instruments, Union City, Calif.) with integrated GenePix 4 software for data acquisition.

\section{Bioinformatics and sequence data handling}

For microarray experiments the raw data of spot fluorescence intensities was collected from slides scanned into GenePix 4 (Axon Instruments, Union City, Calif.). At this stage slide background fluorescence signal was removed from the spot values. The reproducibility of the data within and between slides was examined by performing Pearson correlations of the red and green channels. Slides with correlation coefficients of below 0.96 were rejected from further analysis. Data resulting from repeated experiments on duplicate slides was then input and analysed using GeneSpring 5.1. Initially the median value of the background fluorescence values for the negative control spots was subtracted from all the data points. Any resulting negative values were then set to an arbitrary value of 10 . Subsequently spot values for each slide were linearly scaled using a Perl script to bring the median spot value of every slide to 1,000 . Statistically significant differences within and between the time points were derived from a minimum of 12 replicate slides per sample ( 2 biological replicates $\times 6$ technical replicates) and determined by performing oneway analysis of variance (ANOVA) tests with an individual test $P$ value cutoff of $\leq 0.05$ using a Perl script incorporating a previously described ANOVA module (Orwant et al. 
1999). In order to avoid throwing out interesting genes by applying the overly conservative multiple test correction of multiplying $P$ values by the number of tests performed (nearly 10,000), we chose to investigate further all interesting clones which had a significant ANOVA $P$ value, and showed a fold change of $>2$. We also chose to combine biological and technical replicates to maximise the degrees of freedom available for the ANOVA. This pseudoreplicate approach maximised our ability to detect differential expression within our samples (given that biological and not technical replicates were limiting) at the expense of reducing the applicability of the inferences made to the wider population. Graphical representation and clustered expression analysis output of the significant data was performed using Genespring 5.1.

\section{Real-time RT-PCR}

Two-step real-time, reverse transcription polymerase chain reaction (RT-PCR) experiments were performed using a Roche LightCycler (Roche Diagnostics, Lewes, UK) in order to confirm expression patterns observed from analysis of microarray data. In the first step reverse transcription of samples of $10 \mu \mathrm{g}$ total RNA spiked with $3 \mu \mathrm{l}$ polyadenylated-mRNA control transcript mix was carried out as described by Wilson et al. (2004). After completion the reactions were heat terminated and diluted tenfold with sterile distilled water. Subsequently, using the LightCycler and associated software for data acquisition, 1- $\mu$ l aliquots of each of the diluted RT reactions were subjected to LightCycler capillary (Roche Diagnostics) PCR (1 cycle of $95^{\circ} \mathrm{C}$ for 15 min followed by 45 cycles of $95^{\circ} \mathrm{C}$ for $30 \mathrm{~s}$, then $55^{\circ} \mathrm{C}$ for $30 \mathrm{~s}$ and subsequently $72^{\circ} \mathrm{C}$ for $50 \mathrm{~s}$ ) in the presence of a number of gene-specific forward and reverse primer sets (see Electronic Supplementary Material) and using a QuantiTect SYBR Green PCR kit (Qiagen) as instructed. Fluorescence readings were taken immediately after each extension phase of the PCR process. On completion of the PCR reactions, melting curve and melting point data for individual PCR products was obtained by monitoring the level of fluorescence after each of a series of incremental temperature rises. Finally the purity of the resulting PCR products was analysed by agarose gel elec-

Fig. 1 Tissues from which embryo samples were isolated. Micrographs of the stages between which wheat embryo growth is completed are shown (a 20 days postanthesis, b $26 \mathrm{dpa}$ ). In a, the coleoptile $(c)$ enclosing the primary leaves, the shoot apex $(s a)$, epiblast (ep) and root cap ( $r c$ ) can be clearly seen, and in $\mathbf{b}$ embryo differentiation is complete. Graphical representation of Genespring 5.1 analysed data showing the alteration in transcript accumulation that occurred during the late development, maturation and germination of wheat embryos is also shown. All genes are shown in c and those that significantly (ANOVA $P \leq 0.05$ ) accumulated in $\mathbf{d}$ or declined in e. A scale indicating the relative expression level is provided. For the graphical representations the colour of the lines indicates the relative level of expression of the individual transcripts that occurred at the start of the time course at $21 \mathrm{dpa}$. Pictures of developing and germinating grains are shown below the time points indicated ( $d p a$ days postanthesis, $d p g$ days postgermination) trophoresis. Progress of the PCR reactions was monitored using the associated LightCycler software with PCR product concentrations being determined by comparison with standard reactions performed using human $\beta$-globulin primers and various quantities of human genomic (g) DNA supplied as a standard kit for the LightCycler. The efficiency of the reverse transcription step of the process was determined by monitoring the accumulation of the PCR
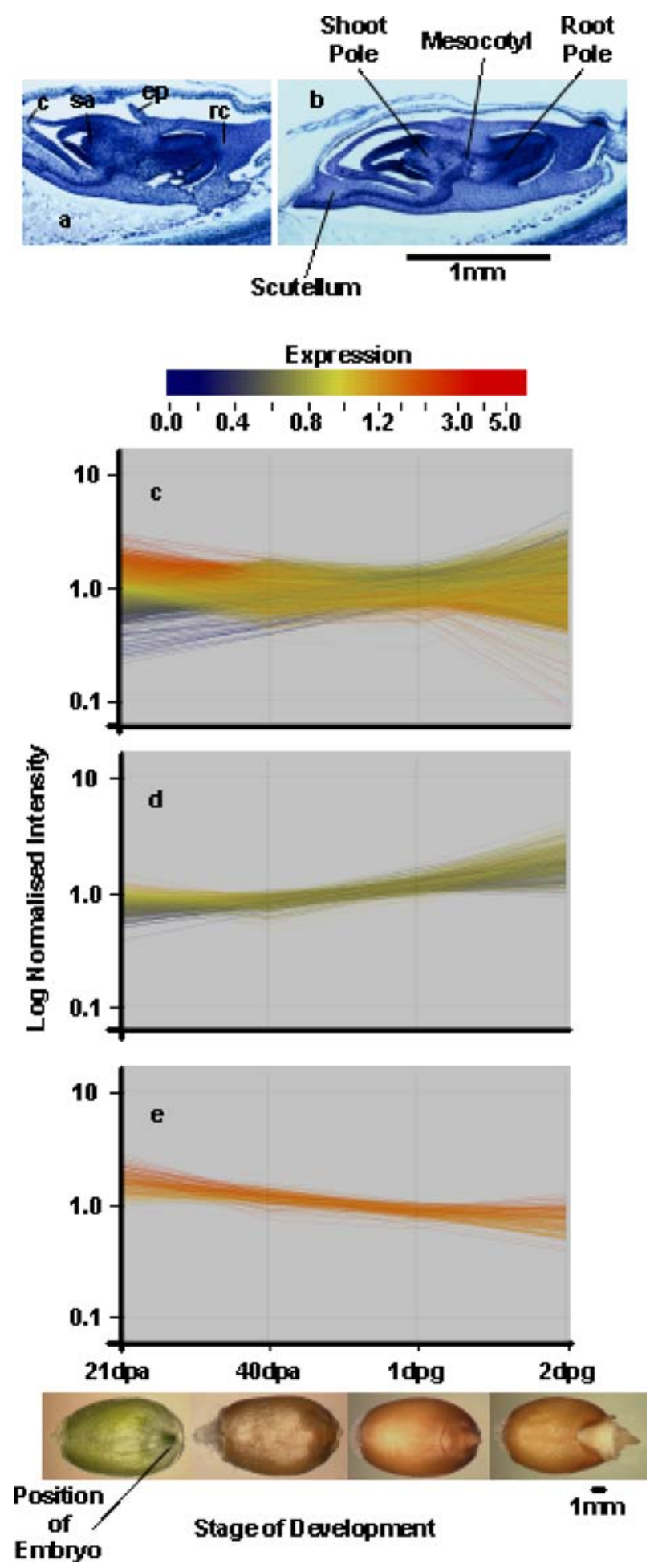
product relating to the TRAF1 mRNA spiked into each of the total RNA samples and adjusting the results accordingly. The absence of gDNA in the RNA samples was also assessed by monitoring the accumulation of any PCR products that accumulated when using the total RNA as a PCR template in reactions using any of the gene-specific primer sets.

\section{Results}

Using microarrays containing 9,155 wheat cDNAs, replicate comparative reverse dye labelling experiments were performed to compare the transcriptomes of late developing and early germinating winter wheat embryos. Assuming that each of the 9,155 cDNAs on the array is capable of hybridising to both its homolog and homeologs, the arrays contain approximately $20 \%$ of the entire wheat genome gene complement. The genes were selected from 35 different cDNA libraries representing a wide range of developmental stages from the early embryo to the seedling stage (Wilson et al. 2004). Here this resource was used to sequentially compare the transcriptomes of late developing embryos at $21 \mathrm{dpa}$ with those that had completed development and desiccated at $40 \mathrm{dpa}$, then $40 \mathrm{dpa}$ embryos with germinating embryos 1 day after imbibition and finally germinating embryos at 1 day postimbibition with those at 2 days postimbibition (Fig. 1). Raw data of spot fluorescence intensities were collected from slides scanned into GenePix 4 and can be viewed as Electronic Supplementary Material. Graphical representation and clustered expression analysis output of the significant data were then performed using GeneSpring 5.1. Using this approach and with reference to the data generated by the real-time RTPCR experiments the dynamic range of detection was such that transcripts representing as little as $1 \times 10^{-6} \%$ of the polyA + mRNA were detectable when using $100 \mu \mathrm{g}$ total RNA to generate fluor-labelled probes and assuming the polyA+ fraction constituted $2.5 \%$ of the total. With the provision that only transcripts showing at least a twofold increase in accumulation level over the time course were of interest, a number of broad patterns of transcript accumulation were apparent during late embryo development and desiccation through to early germination. It was apparent

Fig. 2 Examples of Genespring 5.1 analysed data showing clustering and graphical representation of transcripts that significantly (ANOVA $P \leq 0.05$ ) accumulated or declined during the late development, maturation and germination of wheat embryos. a Cluster analysis of all the transcripts that significantly accumulated (al) or declined ( $a 2)$ over the time course described. b, c Cluster analysis $(b 1$, $c 1)$ and graphical representation $(b 2, c 2)$ of transcripts encoding proteins involved in cell signalling (b) and protein turnover (c) that significantly accumulated over the time course described. d Similar cluster analysis $(d l)$ and graphical representation $(d 2)$ of transcripts encoding proteins involved in cell division that declined during the time course shown. A scale indicating the relative expression level is provided. For each of the cluster analyses a measurement of relative accumulation at each time point is shown. For the graphical representations the colour of the lines indicates the relative level of expression of the individual transcripts that occurred at the start of the time course at $21 \mathrm{dpa}$ that the majority of hybridising transcripts were present at similar levels in all developmental stages of the time course (Fig. 1). For these transcripts there was a small but insignificant fluctuation in overall accumulation levels over time. More apparently there were groups of transcripts that showed lower levels of accumulation during late embryo development and desiccation and then significantly increased over the first $48 \mathrm{~h}$ of germination (Fig. 1). Some 392 sequences in total passed the ANOVA filter and showed

A

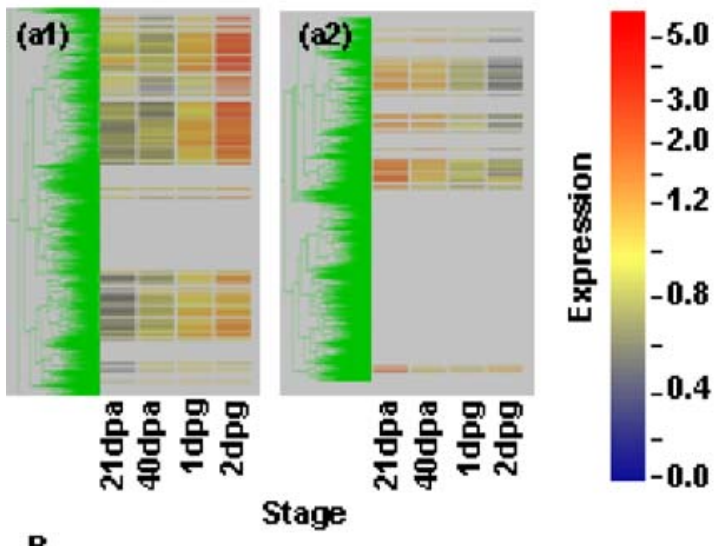

B
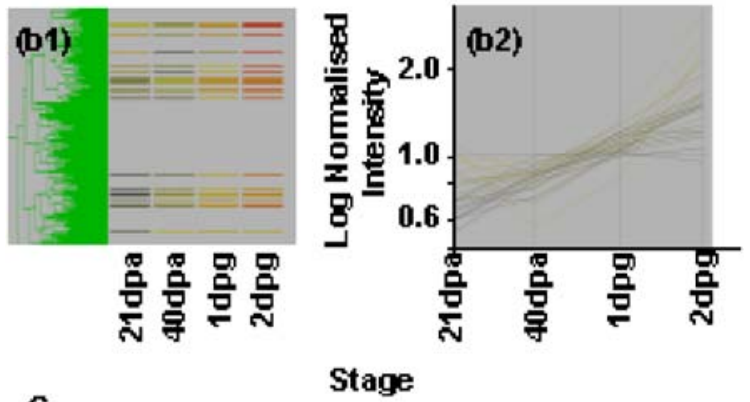

C
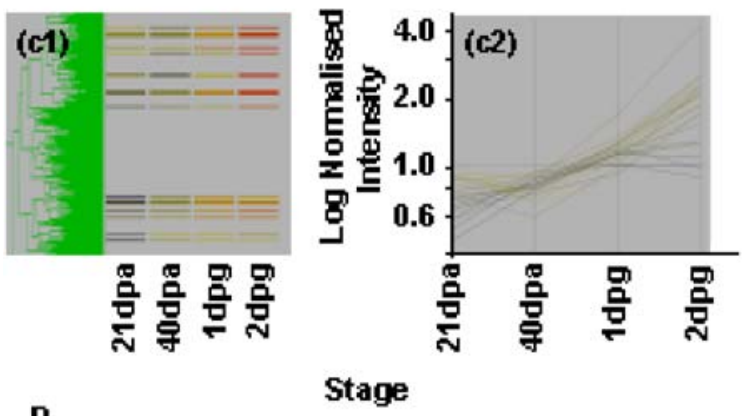

D
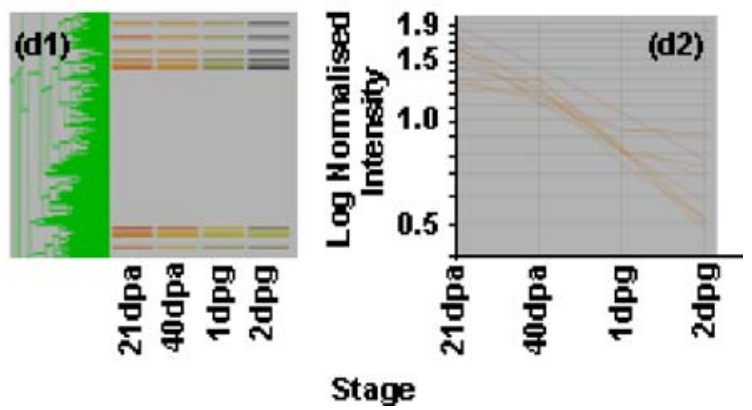
an increase of twofold or greater from $21 \mathrm{dpa}$ to $2 \mathrm{dpg}$. Of these $224(57.1 \%)$ were similar (BLASTX/BLASTN) to annotated sequences encoding proteins of known function, while 168 sequences $(42.9 \%)$ were similar to sequences without functional annotation. These are listed along with their probable functional identities, where known, in
Table S1 (Electronic Supplementary Material). They include genes involved in amino acid biosynthesis and metabolism, cell division and subsequent development, signal transduction, lipid metabolism, energy production, protein turnover, respiration, initiation of transcription, initiation of translation and ribosomal composition. Conversely, other
Fig. 3 Array-determined accumulation patterns for wheat mRNAs encoding proteins similar to: a oat Viviparous-interacting protein 1 , e IAA-Ala hydrolase, f IAA-Ala resistance protein. Bars show the $95 \%$ confidence interval. Real-time RT-PCR-determined accumulation patterns for transcripts encoding: b wheat Viviparous 1 (WVP1) and Viviparous 14 (WVP14), c wheat Viviparousinteracting protein 1 (WVIPl) and Viviparous-interacting protein 2 (WVIP2), d wheat Viviparous-interacting protein 3 (WVIP3), g IAA-Ala hydrolase and IAA-Ala resistance protein are also shown. For the real-time RT-PCR experiments the data shown is the mean of experiments performed on replicates of RNA samples obtained from experiments repeated to obtain duplicate sample material. Primer set sequences for the real-time RT-PCR experiments can be found in the Electronic Supplementary Material
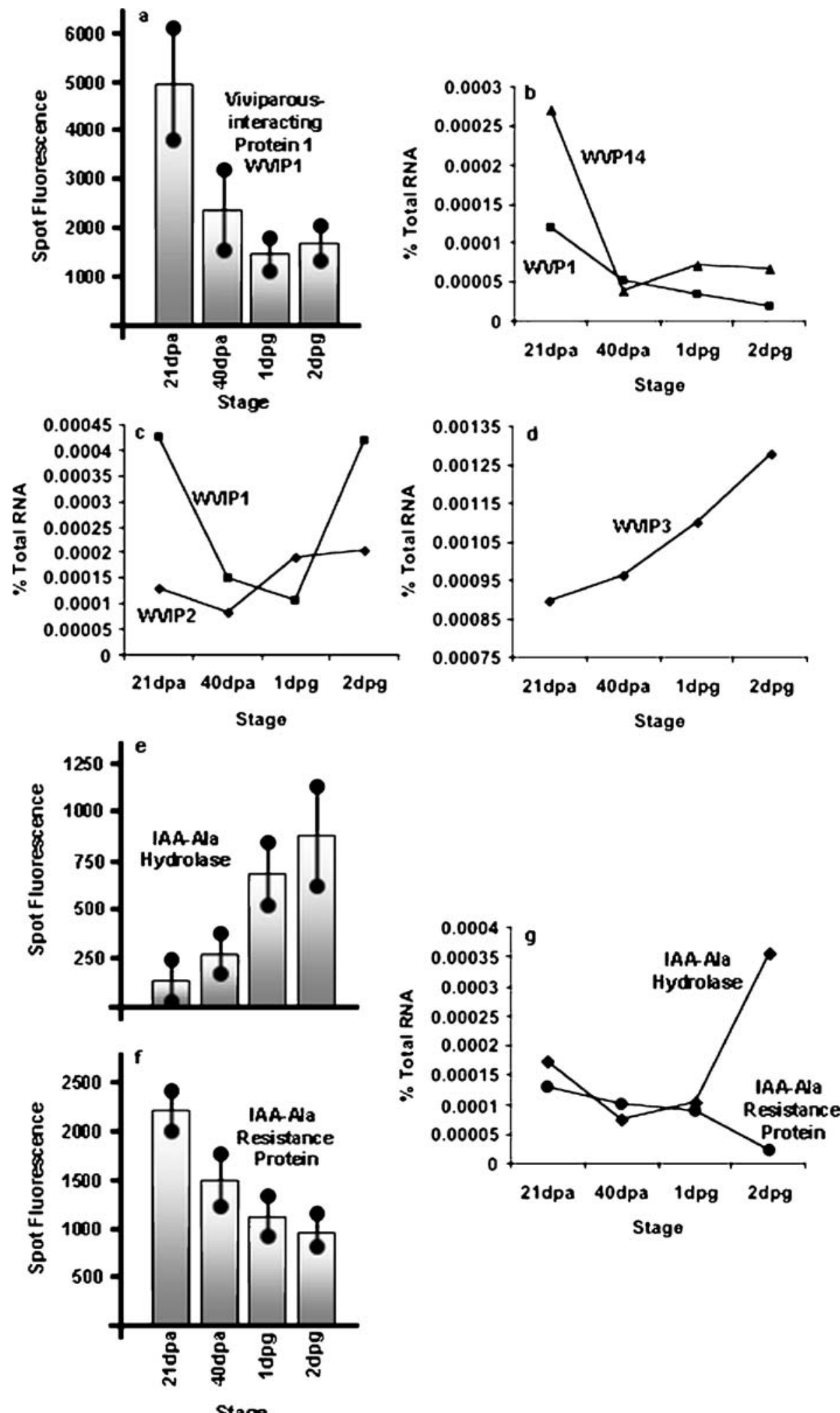
groups of transcripts had relatively high levels of accumulation during late embryo development and desiccation, but decreased during early embryo germination. Some 163 sequences in total showed decreases of twofold or greater over this time course. Of these $83(50.9 \%)$ were similar (BLASTX/BLASTN) to annotated sequences encoding proteins of known function, while $80(49.1 \%)$ were similar to sequences without functional annotation. These sequences are similarly listed in Table S2 (Electronic Supplementary Material). An example of GeneSpring 5.1 clustering analysis of functionally related groups of sequences that either accumulated or declined over the time course is shown in Fig. 2.

A comparison was also made between the ESTs reported here and those identified by macroarray analysis of mRNA accumulation in the embryos of germinating barley grain by Potokina et al. (2002). Of the 52 barley sequences described as expressed in the germinating embryo, here array expression data was available for 36 similarly annotated wheat clones. Of these 36 wheat clones, 21 showed a similar pattern of expression to their barley counterparts. However, there were differences in the absolute levels of accumulation detected and the overall fold changes observed. Generally the fold changes observed here were lower than those described for barley.

The protein Vp1 has been shown to have an important role in regulating germination. Thus, we also identified transcripts with altered accumulation patterns that encoded proteins associating with Vp1. Recently, there has been renewed focus on the role of auxin in germination. Therefore, we also identified mRNAs with altered patterns of accumulation during germination that encoded proteins involved in determining sensitivity to the inhibitory effects of conjugated auxin and the levels of active unconjugated auxin. Thus, graphical representation of the array-determined patterns of accumulation for mRNAs encoding proteins with similarities to the oat Viviparous-interacting protein 1 , rice IAA-Ala hydrolase and $A$. thaliana IAA-Ala resistance protein are also shown in Fig. 3. Transcripts encoding WVIP1 appeared to decline between 21 dpa until $1 \mathrm{dpg}$ and thereafter began to accumulate again at $2 \mathrm{dpg}$. Transcripts encoding IAA-Ala hydrolase appeared to accumulate over the time course from $21 \mathrm{dpa}$ to $2 \mathrm{dpg}$ while those encoding the IAA-Ala resistance protein declined throughout. In addition the expression pattern of wheat homologs of $\mathrm{Vp} 1$ and other transcripts encoding protein that are known to interact with this protein were also determined by quantitative real-time RT-PCR (Fig. 3). Apart from WVIP1, cDNAs encoding these proteins were not represented on the array, but in light of the results obtained for WVIP1 it was deemed prudent to similarly examine the accumulation patterns of these mRNAs. Transcripts encoding WVIP1 showed a similar pattern of accumulation to that observed from analysis of the array data, but the increased accumulation at $2 \mathrm{dpg}$ was more obvious. The determined patterns of accumulation of transcripts encoding IAA-Ala hydrolase and the IAA-Ala resistance protein were generally similar to those determined from the array data in that those for the IAA-Ala hydrolase accumulated over the time course while those for the IAAAla resistance protein declined. However, here instead of gradually increasing between each time point as suggested by the array data, the transcripts encoding the IAA-Ala hydrolase declined between 21 and 40 dpa before increasing to a maximum level over the first $48 \mathrm{~h}$ of germination. Quantitative real-time RT-PCR also determined that the levels of transcripts encoding wheat Viviparous-interacting protein 2 and 3 (WVIP2 and WVIP3) were elevated in the embryos of germinating grains when compared to those of late maturing and desiccated grains. Transcripts encoding wheat Viviparous 1 (WVP1) appeared to decline throughout the time course from $21 \mathrm{dpa}$ to $2 \mathrm{dpg}$. Those encoding wheat VP14 (WVP14) appeared to decline between 21 and $40 \mathrm{dpa}$ and then showed a small increase again as germination progressed.

In order to confirm the observed trends in mRNA accumulation determined from array data, real-time RT-PCR experiments were performed on a selection of individual clones. A summary of all the results of these real-time RTPCR experiments is given in Table S3 (Electronic Supplementary Material). Fourteen of the 17 sequences tested by quantitative real-time RT-PCR produced data of the same sign and within one order of magnitude of that resulting from the array data. Of these, 12 were different from the array data by no more than threefold. Thus, approximately $70 \%$ of the real-time RT-PCR experiments confirmed observations made from the analysis of the array data.

\section{Discussion}

Changes in the transcriptome of maturing and germinating wheat embryos

We have used cDNA microarrays to investigate alterations in the transcriptome of wheat embryos during the last stages of maturation and dry down and on into early germination and have used real-time RT-PCR to confirm that approximately $70 \%$ of the observations made are accurate with regard to the indicated direction of accumulation of the transcripts. This level of determined accuracy fell generally within the findings of other groups undertaking similar studies (for an overview see Wurmbach et al. 2003) and, in addition to all the other sources of variation inherent to microarray experiments (Chuaqui et al. 2002), most likely reflected the differences characteristic of each of the expression analysis methods. For instance cDNA arrays such as those used here suffer in their inability to differentiate between the hybridisation of closely related sequences (homologs, homeologs and paralogs), while the use of gene-specific primers for real-time RT-PCR analysis is much more discriminating. In addition, here the process of normalising the array data assumed that the level of polyA + mRNA had remained constant as a percentage of the total RNA in each sample. The real-time RT-PCR experiments on the other hand measured absolute levels. Although an in-depth assessment of polyA+ mRNA levels in each of the samples was not carried out here, it was 
obvious that the level of extractable total RNA and the percentage recovery of polyA + mRNA from total RNA was always lower in the mature, dormant embryo samples. This would inevitably have resulted in the array data analysis providing an overstatement of the absolute levels of any particular mRNA in this sample compared to the others. Thus, the discrepancies between expression levels determined by array hybridisations and those observed by RT-PCR may not have been unexpected in this case. The results described here are considerably more comprehensive than those previously described for barley by Potokina et al. (2002). However, where the data is duplicated the results appear to be in general agreement. The differences in expression patterns or levels of mRNA accumulation that were apparent between wheat and barley probably reflected the different technologies used in each case. Thus, we suggest that the alterations of the transcriptome described here are consistent with the biology of seed germination and, therefore, that the novel association of the large number of transcripts encoding proteins of unknown function with this process are both real and significant.

Changes in accumulation of mRNAs encoding proteins involved in amino acid biosynthesis

A number of mRNAs encoding proteins involved in amino acid biosynthesis, metabolism and transport showed increased levels of accumulation over the time course outlined. This included the sequence encoding methionine synthase which has been shown to be important for seed germination in Arabidopsis (Gallardo et al. 2002b). Changes in the expression of genes involved in amino acid synthesis and metabolism have been similarly reported during germination in barley (Potokina et al. 2002) and Vicia faba (Miranda et al. 2003). During the germination of Medicago trunculata it was reported that the accumulation of mRNAs encoding glutamine synthase 2 and glutamate dehydrogenase was significantly increased in the embryonic axis within $48 \mathrm{~h}$ (Glevarec et al. 2004) postimbibition. The changes observed here appeared less dramatic than this with no large alteration in the accumulation of mRNAs encoding enzymes involved in the GS/GOGAT pathway during the time course described. Here glutamine synthase mRNA was present at reasonable levels throughout the experiment with a small increase at the 2-dpg time point. Similarly the mRNA encoding glutamate dehydrogenase was expressed throughout, but declined slightly at the onset of germination. Whether or not these differences simply reflected differences in the rate of mobilisation of stored nitrogen reserves in the monocot or whether they reflected a more fundamental deviation in the underlying mechanisms of germination in the monocots resulting from their different physiology is unknown. However, in maize the efficiency of germination has been linked to the rate at which stored nitrogen can be converted to glutamine by glutamine synthase (Limami et al. 2002). Also, the changes in the levels of the mRNAs described here may not accurately reflect the protein levels in the embryo. Thus, it may be that wheat embryos use more stored mRNAs while those of Medicago achieve the same goal by de novo synthesis during the early stages of germination.

Changes in accumulation of mRNAs encoding proteins involved in cell division and the formation of new cells and cell walls

As may have been expected, the first $48 \mathrm{~h}$ of germination were accompanied by increases in the levels of many mRNAs encoding proteins involved in the formation of new cells. For instance we observed increases from a basal level in mRNAs encoding cytoskeletal components such as myosin and actin, cell division proteins such as cyclindependent kinase $\mathrm{C}$ and proteins such as histone $2 \mathrm{~A}$ and $2 \mathrm{~B}$ which are involved in chromatin formation. Such observations were in accordance with the processes known to occur in active meristematic tissues. There were also a number of mRNAs encoding carbohydrate metabolising enzymes that accumulated in the embryo during germination. Some of these, such as that encoding cellulose synthase, are obviously involved in the synthesis of new cell walls. However, the exact role of some of the other carbohydrate metabolising enzymes is unclear. For example $\beta$-D-xylosidase has been purified from barley grains and has been shown to be active in the hydrolysis of oligosaccharide products released from wheat flour arabinoxylan by the activity of (1-4)- $\beta$-D-xylan endohydrolase (Lee et al. 2003). However, the mRNA encoding this enzyme was also shown to be present in most barley tissues. Thus, there was some difficulty in assigning a precise role for this enzyme in either cell wall formation or the hydrolysis of stored reserves. The absence of accumulation of mRNA encoding $\alpha$-amylase in the dataset would indicate that the isolated embryos were pure, uncontaminated with cells from the aleurone layer. However, enzymes such as $\alpha$ glucosidase are known to be involved in starch metabolism (Van der Maarel et al. 2002). Thus, the inference here was that the embryos accumulated mRNAs encoding starch metabolising enzymes which acted on the endosperm reserves during germination. In dicots such enzymes have been shown to be induced by GA in the germinating embryo in order to digest the surrounding endosperm prior to emergence of the radicle (Ogawa et al. 2003). In wheat where the radicle simply emerges through the dead seed coat the argument is less clear. Perhaps in wheat such enzymes are produced by the embryo simply to aid in mobilisation of stored reserves in the endosperm.

Changes in accumulation of mRNAs encoding proteins involved in respiration and the production of energy

Respiration and the production of energy are processes which resume very rapidly following imbibition of dormant seeds (Botha et al. 1992; Bewley and Black 1994). Here, the accumulation of mRNAs encoding key enzymes 
involved in both the glycolytic and tricarboxylic acid (TCA) cycle pathways was increased in the germinating wheat embryos. In addition the mRNA encoding fructose1-6-bisphosphatase, an enzyme involved in the conversion of fructose-1-6-bisphosphate to fructose-6-phosphate as part of the Calvin cycle, also showed elevated levels during embryo germination. Such increases indicated the utilisation of stored carbohydrates and increased input to the TCA cycle along with increased ability to restore $\mathrm{CO}_{2}$ fixation. In particular the dramatic rise in the level of aldolase, an enzyme which converts fructose-1-6-bisphosphate into dihydroxyacetone phosphate and glyceraldehyde-3-phosphate, at the onset of germination indicated a sudden and rapid entry of hexose sugars into the glycolytic pathway. Such phenomena are entirely commensurate with the processes of mobilisation and use of carbohydrates stored in the endosperm. Interestingly the levels of mRNAs encoding the regulatory proteins of glycolysis, namely hexokinase, phosphofructokinase and pyruvate kinase, did not alter significantly during the germination of the embryos. A similar finding has been described for barley phosphofructokinase during embryo germination (Potokina et al. 2002). Possibly the control of glycolytic flux by phosphofructokinase during early germination is regulated allostearically by low levels of ATP and citrate which would promote activity of the enzyme as a result of their diminished inhibitory effects on its affinity for fructose-6phosphate. However, the mRNA encoding this enzyme was present at reasonably high and similar levels prior to and during the germination period. Thus, it is tempting to suggest that prior to germination the mature embryo contained sufficient transcripts encoding the regulatory enzymes of glycolysis such that on imbibition and restoration of translation the resulting levels of these enzymes were not limiting on the ability to utilise hexose sugars in the glycolytic pathway.

Changes in accumulation of mRNAs encoding proteins involved in the mobilisation of stored proteins

In the dormant seed, stored proteins that are mobilised during germination are contained within protein storage bodies which have been found in the embryos of a number of plants species (Tiedemann et al. 2000). Endopeptidases and carboxypeptidases are also present in the protein bodies of the dry seed, but there is still debate concerning their role in the mobilisation of stored proteins during germination (for review see Muntz et al. 2001). In line with most reviews the results presented here have provided further evidence that the mobilisation of stored proteins during germination involves increased gene expression and the resulting production of increased levels of proteinases. However, it is clear that transcripts encoding these proteolytic enzymes were present in wheat embryos prior to and at the point of desiccation. Thus, the results also provide fuel to the argument that these enzymes exist in the dry embryo and have a role to play in initiating the mobilisation of stored proteins during the onset of germination.
The accumulation during germination of a mRNA encoding a proteinase inhibitor also suggests that the activity of some preexisting proteinases may be inhibited during this process or that anabolic processes pertaining to the synthesis of new cells require a degree of protection in a proteolytic environment.

Are there germination-specific mRNAs in wheat?

Germination constitutes a dramatic developmental change in the physiological state of the embryo. The results presented here were clearly indicative of a rapid mobilisation of storage reserves and the synthesis of new cells, intercellular structures and new proteins along with the required attendant rise in respiration and energy production. There was also evidence of differential cell signalling and the synthesis of new enzymes. In such circumstances one would possibly have expected considerable alteration in the composition of the embryo transcriptome. However, with wheat this study does not appear to support such a case. The total number of sequences that demonstrated significant alteration in level over the time course described here was relatively small and only a few of these sequences could be described as possibly following a pattern of germination-specific accumulation or decline. In fact the majority of the transcripts that did appear to accumulate in the embryo from late development (21 dpa) through to the second day of germination were present in the mature, dormant embryo (40 dpa) and early germinating embryo (1 dpg) at quite similar, often insignificantly different levels. In other words, although many transcripts showed an overall change of greater than twofold over the whole time course, for many of these transcripts the degree of change between each successive time point was relatively small. Thus, it would appear that the cells of the mature, dormant embryo accumulated the vast majority of the transcripts encoding the proteins required to continue metabolism following the initiation of germination. Other recent studies (Girke et al. 2000; Gallardo et al. 2001) have described similar findings that add support to the suggestion that the mature embryo of the dormant seed can be regarded as a resting miniature plantlet that contains the majority of the transcripts required for continued growth on reawakening (Bewley 1997). The majority of the 1,251 proteins examined in germinating Arabidopsis embryos by Gallardo et al. (2001) were also found in the dormant embryo. It is also recognised that the translation machinery reforms rapidly following imbibition (Dommes and Van der Walles 1990). Here the observed increase in accumulation prior to and during germination of transcripts encoding translation activation factors supports this concept. The suggestion would be that the rapid translation of existing transcripts is the predominant mechanism whereby the processes of early germination get underway. Thus, the initial break from dormancy into germination may only involve the synthesis of a very few new transcripts. However, it is clear that the first $48 \mathrm{~h}$ of wheat germination did involve the transcription of some new mRNAs and a 
decline in the level of others. The accumulation of transcripts encoding the transcription initiation factors IIA and IIB during germination suggested a process of generally increasing transcription. We have also reported a small number of transcripts that either accumulated or declined significantly only after germination had begun. In the main those that accumulated in this manner encoded various proteins of unknown function, but also included transcripts encoding a number of RNA binding proteins, quinone oxidoreductase, chloroplastic aldolase, various transcription factors and a protein similar to the tomato SKIP5 protein which interacts with the protein SKP1 (Farras et al. 2001), a protein that connects cell cycle regulators to the ubiquitin proteolysis machinery through an F-box domain (Bai et al. 1996). The question is whether or not any of these transcripts were directly involved in germination per se or were, alternatively, involved in postgermination events associated with seedling growth and development. The current consensus of opinion is that the latter case is the more likely and that these transcripts were synthesised de novo once germination had started (Bewley 1997; Bove et al. 2001). In this study the transcripts that declined significantly at the onset of germination included a number encoding proteins of unknown function, those encoding proteins associated with the storage of reserves and those encoding inhibitors such as the $\alpha$-tubulin suppressor protein which may inhibit some of the processes involved in germination. Again, it is not known whether any of the declining transcripts that encoded proteins of unknown function were directly involved in the initiation of or in the early events of germination. However, these sequences do represent possible targets for future investigation.

It would, therefore, appear that the very nature of dormancy and the ability of the dormant embryo to store mRNA make for a degree of difficulty in determining the importance of individual genes when using a temporally correlative transcriptomics approach. However, this approach has resulted in a number of candidates for future study. For example, in addition to the small number of genes encoding proteins of unknown function that may be involved in germination, in this study a transcript (WVIP1) encoding a protein similar to oat Viviparous- (Vp) interacting protein 1 (AfVIP1; Jones et al. 2000) declined significantly as the embryo reached maturity, reached a minimum level during the first $24 \mathrm{~h}$ of germination and then returned to previous levels by $2 \mathrm{dpg}$ (Fig. 3a, c). The function of this protein is unknown and it is not known if the transcript level reflected levels of protein accumulation here, but the known interaction of this protein with Vp1 adds weight to the suggestion that alterations in the abundance of its encoding transcript just prior to germination are important. The patterns of accumulation during grain development and germination of transcripts encoding WVIP1 and those encoding wheat homologs (WVP1 and WVIP3) of the oat Viviparous 1 and Viviparous-interacting protein 3 were reasonably consistent with those described previously (Jones et al. 2000). Our results confirm that levels of transcripts encoding WVIP1 (Fig. 3a, c) and WVIP3 (Fig. 3d) are elevated in germinating embryos as compared to those of the grain before imbibition and that transcripts encoding WVP1 (Fig. 3b) generally declined over time. Our results also show that transcripts encoding WVIP1 are at elevated levels in the developing embryo at the stage of completion of differentiation and that the decline in the level of this transcript observed as the embryo desiccated, persisted until $24 \mathrm{~h}$ after germination had begun (Fig. 3c). The implications of this pattern of accumulation remain unclear. However, it is intriguing that of all the transcripts encoding Viviparous-interacting proteins those encoding WVIP1 followed a pattern inverse to the process of germination. Perhaps the interaction of WVIP1 with Viviparous 1 enhanced the effect of ABA signalling. Thus, germination may have been potentiated by a reduction of this interaction just prior to and during the first $24 \mathrm{~h}$ of this process. The level of accumulation of transcripts encoding WVP14, a wheat homolog of maize VP14 which encodes a protein that cleaves 9-cis-epoxy-carotenoids to form $\mathrm{C}_{25}$ apo-aldehydes and xanthoxin, a precursor of ABA (Schwartz et al. 1997, 2003), also declined prior to germination (Fig. 3b). Thus, there may have been a reduction in potential ABA synthesis coincident with reduced ABA sensitivity just prior to wheat germination. Transcripts encoding the wheat homolog (WVIP3) of oat AfVIP3 encoded a protein with similarities to a human cell crisis-related protein with possible transcription factor activity at the $\mathrm{G}_{1}$-S phase transition of the cell cycle (Ilijima et al. 1996). One suggestion that has been made is that the interaction of AfVIP3 protein with Vp1 may modulate its activity and act to maintain the embryo in the dormant $\mathrm{G}_{1}$ phase state (Jones et al. 2000). However, transcripts encoding WVIP3 accumulated during germination while those encoding WVP1 declined. Also, although it is unknown if transcript levels reflected final protein levels, the levels of transcripts encoding WVIP3 were always considerably higher than those encoding WVP1. The rise in the level of transcripts encoding WVIP3 during germination certainly coincided with the appearance of other transcripts encoding proteins involved in cell division and the formation of new cells and cytoskeleton. Thus, if WVIP3 promoted the $\mathrm{G}_{1}-\mathrm{S}$ phase transition associated with the cell division that accompanies germination, we would suggest that its interaction with WVP1 may have acted to remove relevant aspects of the repression on germination. The pattern of accumulation of transcripts encoding a wheat homolog (WVIP2) of oat Viviparous-interacting protein 2 (AfVIP2) was not consistent with that reported previously, where the level of accumulation remained similar in dormant and germinating seeds (Jones et al. 2000). Our results suggest elevated levels of transcripts encoding WVIP2 occurred in germinating embryos (Fig. 3c). Perhaps this reflects the specific use of embryo rather than whole grain tissues in our study, but it might also suggest subtle differences between the mechanisms of germination of the different cereals. The protein AfVIP2 contains a ring $\left(\mathrm{C}_{3} \mathrm{HC}_{3}\right.$-type $)$ zinc finger proteinprotein interaction domain (Meshi and Iwabuchi 1995) near to the N-terminus. Many proteins containing this domain are involved in transcriptional regulation (Freemont 1993). How the interaction of this protein modulates the ABA 
signalling activity of $\mathrm{Vp} 1$ remains unclear. However, a rise in the level of its encoding transcript after germination is interesting. Possibly WVIP2 also interacted with ABAresponsive elements in a manner analogous to that observed for rice TRAB1 (Hobo et al. 1999) which appears to bridge the gap between $\mathrm{Vp} 1$ and these DNA elements or perhaps WVIP2 modulated the interaction of a protein similar to TRAB1 with Vp1. Whichever, such hypotheses warrant further investigation.

Our results showing the decline during maturation and germination of a transcript encoding a protein similar to $A$. thaliana IAA-Ala resistance protein 1 (Lasswell et al. 2000) and the coincident rise in a transcript encoding a protein with similarity to rice IAA-Ala hydrolase suggest that the level of free, unconjugated, active auxin may be associated with postgermination coleoptile expansion. However, recent studies have also implicated an inhibitory role of auxin in the germination of wheat grains (Ramaih et al. 2003). The suggestion was that auxin may complement the role of $\mathrm{ABA}$ in regulating dormancy and the onset of germination. However, there was no measurement of the degree of conjugation of the applied auxin in these studies. Thus, the possibility that a balance between the levels of conjugated and free auxin plays a role in germination remains relevant. Increased levels of transcripts encoding the auxin-binding protein 1 in the meristems of germinating sunflower seeds (Thomas et al. 2003) would indicate that responses to free auxin play a role in the germination of these tissues. However, the exact nature of the role of auxin in wheat germination awaits more precise investigation.

\section{Conclusion}

This paper presents the first report of the use of a publicly available transcriptomics resource for wheat and details the first large-scale study of the alteration of the wheat embryo transcriptome during maturation and germination. We have identified a number of genes which may have important bearing on these processes and with the availability of cereal transgenic and knockout systems in rice and maize future work may be able to assess their functional importance. In addition it should be possible to use the rice genome sequence to putative map the several hundred sequences identified in this work to see if they map to regions thought to be associated with QTLs for dormancy and/or germination. If they do such sequences may serve as genetic markers which could be used to distinguish between varieties with different propensities for precocious germination. Such markers could also be of use in the development of new cereal varieties with superior dormancy or germination traits. As with other plants we have implicated alterations in ABA and possibly auxin signalling in this transition from the dormant to germinating state. In addition to wheat this has obvious implications for those with interests in the process of germination in other cereals. For example, the discovery of new genes important to the malting process in barley may be hampered by the lack of differential gene and protein expression between the ma- ture dormant and germinating embryo. Presumably many sequences encoding proteins important to germination begin to accumulate during maturation soon after differentiation of the embryo and before dormancy. Thus, this earlier time period warrants further and more detailed investigation and here the transcriptomics approach may have considerable value. Bearing in mind the sequence divergence between the monocots and dicots it is inevitable that the application of directed mutation programmes will also be a requirement for a more complete understanding of germination in the cereals.

Acknowledgements This work was supported by the Biotechnology and Biological Sciences Research Council, UK (BBSRC) under the cereals "Investigating Gene Function" initiative (ref. IGF12403). Chungui Lu was funded by a BBSRC Agri-Food response mode grant to investigate the effect of nitrogen supply on the wheat endosperm transcriptome (ref. D16781). Our thanks to Bob Hughes, Richard Parkinson and Derek Edgell of the now closed Long Ashton Research Station, Long Ashton, Bristol, UK, for their invaluable assistance with controlled environment growth of wheat plants. We are also grateful to Drs Steve White and Mark Bond of the Bristol Royal Infirmary, Bristol BS2 8HW, UK, for their assistance with real-time RT-PCR experiments.

\section{References}

Agrawal GK, Yamazaki M, Kobayashi M, Hirochika R, Miyao A, Hirochika H (2001) Screening of the rice viviparous mutants generated by endogenous retrotransposon Tos 17 insertion. Tagging of a zeaxanthin epoxidase gene and a novel ostatc gene. Plant Physiol 125(3):1248-1257

Bai C, Sen P, Hofmann K, Ma L, Goebl M, Harper JW, Elledge SJ (1996) SKP1 connects cell cycle regulators to the ubiquitin proteolysis machinery through a novel motif, the F-box. Cell 86 (2):263-274

Bailey PC, McKibbin RS, Lenton JR, Holdsworth MJ, Flintham JE, Gale MD (1999) Genetic map locations for orthologous Vp1 genes in wheat and rice. Theor Appl Genet 98:281-284

Bewley JD (1997) Seed germination and dormancy. Plant Cell 9 (7):1055-1066

Bewley JD, Black M (1994) Seeds: physiology of development and germination. Plenum, New York

Botha FC, Potgieter GP, Botha AM (1992) Respiratory metabolism and gene expression during seed germination. J Plant Growth Regul 11:211-224

Bove J, Jullien M, Grappin P (2001) Functional genomics in the study of seed germination. Genome Biol 3(1):1-5

Chen GP, Wilson ID, Kim SH, Grierson D (2001) Inhibiting expression of a tomato ripening-associated membrane protein increases organic acids and reduces sugar levels of fruit. Planta 212(5-6):799-807

Chuaqui RF, Bonner RF, Best CJ, Gillespie JW, Flaig MJ, Hewitt SM, Phillips JL, Krizman DB, Tangrea MA, Ahram M, Linehan WM, Knezevic V, Emmert-Buck MR (2002) Postanalysis follow-up and validation of microarray experiments. Nat Genet 32(Suppl):509-514

Debeaujon I, Koornneef M (2000) Gibberellin requirement for Arabidopsis seed germination is determined both by testa characteristics and embryonic abscisic acid. Plant Physiol 122:415-424

Dommes J, Van der Walles C (1990) Polysome formation and incorporation of new ribosomes into polysomes during germination of the embryonic axis of maize. Physiol Plant 79:289-296

Farras R, Ferrando A, Jasik J, Kleinow T, Okresz L, Tiburcio A, Salchert K, del Pozo C, Schell J, Koncz C (2001) SKP1-SnRK protein kinase interactions mediate proteasomal binding of a plant SCF ubiquitin ligase. EMBO J 20(11):2742-2756 
Finkelstein RR, Zeevaart JAD (1994) Gibberellin and abscisic acid biosynthesis and response. In: Somerville C, Meyerowitz E (eds) Arabidopsis Cold Spring Harbor Laboratory Press, Cold Spring Harbor, N.Y.

Freemont PS (1993) The RING finger. A novel protein sequence motif related to the zinc finger. Ann NY Acad Sci 684:174-192

Gallardo K, Job C, Groot SP, Puype M, Demol H, Vandekerckhove J, Job D (2001) Proteomic analysis of Arabidopsis seed germination and priming. Plant Physiol 126(2):835-848

Gallardo K, Job C, Groot SP, Puype M, Demol H, Vandekerckhove J, Job D (2002a) Proteomics of Arabidopsis seed germination. A comparative study of wild-type and gibberellin-deficient seeds. Plant Physiol 129(2):823-837

Gallardo K, Job C, Groot SP, Puype M, Demol H, Vandekerckhove J, Job D (2002b) Importance of methionine biosynthesis for Arabidopsis seed germination and seedling growth. Physiol Plant 116(2):238-247

Giraudat J, Hauge BM, Valon C, Smalle J, Parcy F, Goodman HM (1992) Isolation of the Arabidopsis ABI3 gene by positional cloning. Plant Cell 4(10):1251-1261

Girke T, Todd J, Ruuska S, White J, Benning C, Ohlrogge J (2000) Microarray analysis of developing Arabidopsis seeds. Plant Physiol 124(4):1570-1581

Glevarec G, Bouton S, Jaspard E, Riou MT, Cliquet JB, Suzuki A, Limami AM (2004) Respective roles of the glutamine synthetase/glutamate synthase cycle and glutamate dehydrogenase in ammonium and amino acid metabolism during germination and post-germinative growth in the model legume Medicago truncatula. Planta 219(2):286-297

Hattori T, Terada T, Hamasuna ST (1994) Sequence and functional analyses of the rice gene homologous to the maize Vp1. Plant Mol Biol 24(5):805-810

Himi E, Mares DJ, Yanagisawa A, Noda K (2002) Effect of grain colour gene (R) on grain dormancy and sensitivity of the embryo to abscisic acid (ABA) in wheat. J Exp Bot 53(374):1569-1574

Hobo T, Kowyama Y, Hattori T (1999) A bZIP factor, TRAB1, interacts with VP1 and mediates abscisic acid-induced transcription. Proc Natl Acad Sci USA 96(26):15348-15353

Ilijima M, Kano Y, Nohno T, Namba M (1996) Cloning of a cDNA with possible transcription factor activity at the G1-S phase transition in human fibroblast cell lines. Acta Med Okayama 50:73-77

Jones HD, Peters NC, Holdsworth MJ (1997) Genotype and environment interact to control dormancy and differential expression of the Viviparous 1 homologue in embryos of Avena fatua. Plant J 12(4):911-920

Jones HD, Kurup S, Peters NC, Holdsworth MJ (2000) Identification and analysis of proteins that interact with the Avena fatua homologue of the maize transcription factor Viviparous 1. Plant J 21(2):133-142

Kato K, Nakamura W, Tabiki T, Miura H, Sawada S (2001) Detection of loci controlling seed dormancy on group 4 chromosomes of wheat and comparative mapping with rice and barley genomes. Theor Appl Genet 102:980-985

Koornneef M, Reuling G, Karssen C (1984) The isolation and characterization of abscisic acid-insensitive mutants of Arabidopsis thaliana. Physiol Plant 61:377-383

Koornneef M, Bentsink L, Hilhorst H (2002) Seed dormancy and germination. Curr Opin Plant Biol 5(1):33-36

Lasswell J, Rogg LE, Nelson DC, Rongey C, Bartel B (2000) Cloning and characterization of $I A R 1$, a gene required for auxin conjugate sensitivity in Arabidopsis. Plant Cell 12:2395-2408

Lee RC, Hrmova M, Burton RA, Lahnstein J, Fincher GB (2003) Bifunctional family 3 glycoside hydrolases from barley with alpha-L-arabinofuranosidase and beta-D-xylosidase activity. Characterization, primary structures, and $\mathrm{COOH}$-terminal processing. J Biol Chem 278(7):5377-5387

Limami AM, Rouillon C, Glevarec G, Gallais A, Hirel B (2002) Genetic and physiological analysis of germination efficiency in maize in relation to nitrogen metabolism reveals the importance of cytosolic glutamine synthetase. Plant Physiol 130(4):18601870
McCarty DR, Hattori T, Carson CB, Vasil V, Lazar M, Vasil IK (1991) The Viviparous-1 developmental gene of maize encodes a novel transcriptional activator. Cell 66(5):895-905

McKibbin RS, Wilkinson MD, Bailey PC, Flintham JE, Andrew LM, Lazzeri PA, Gale MD, Lenton JR, Holdsworth MJ (2002) Transcripts of $\mathrm{Vp}-1$ homeologues are misspliced in modern wheat and ancestral species. Proc Natl Acad Sci USA 99 (15):10203-10208

Meshi T, Iwabuchi M (1995) Plant transcription factors. Plant Cell Physiol 36(8):1405-1420

Miranda M, Borisjuk L, Tewes A, Dietrich D, Rentsch D, Weber H, Wobus U (2003) Peptide and amino acid transporters are differentially regulated during seed development and germination in faba bean. Plant Physiol 132(4):1950-1960

Muntz K, Belozersky MA, Dunaevsky YE, Schlereth A, Tiedemann J (2001) Stored proteinases and the initiation of storage protein mobilization in seeds during germination and seedling growth. $\mathrm{J}$ Exp Bot 52(362):1741-1752

Nambara E, Keith K, McCourt P, Naito S (1995) A regulatory role for the $A B I 3$ gene in the establishment of embryo maturation in Arabidopsis thaliana. Development 121:629-636

Ogawa M, Hanada A, Yamauchi Y, Kuwahara A, Kamiya Y, Yamaguchi S (2003) Gibberellin biosynthesis and response during Arabidopsis seed germination. Plant Cell 15(7):15911604

Orwant J, Hietaniemi J, Macdonald J (1999) In: Oram A, Orwant J (eds) Mastering algorithms with Perl. O'Reilly, Sebastopol, Calif.

Osa M, Kato K, Mori M, Shindo C, Torada A, Miura H (2003) Mapping QTLs for seed dormancy and the Vp1 homologue on chromosome 3A in wheat. Theor Appl Genet 106(8):1491-1496

Potokina E, Sreenivasulu N, Altschmied L, Michalek W, Graner A (2002) Differential gene expression during seed germination in barley (Hordeum vulgare L.). Funct Integr Genomics 2(1-2): 28-39

Ramaih S, Guedira M, Paulsen GM (2003) Relationship of indoleacetic acid and tryptophan to dormancy and preharvest sprouting of wheat. Funct Plant Biol 30(9):939-945

Schwartz SH, Tan BC, Gage DA, Zeevaart JA, McCarty DR (1997) Specific oxidative cleavage of carotenoids by VP14 of maize. Science 276(5320):1872-1874

Schwartz SH, Tan BC, McCarty DR, Welch W, Zeevaart JA (2003) Substrate specificity and kinetics for VP14, a carotenoid cleavage dioxygenase in the ABA biosynthetic pathway. Biochim Biophys Acta 1619(1):9-14

Suzuki M, Kao CY, McCarty DR (1997) The conserved B3 domain of Viviparous1 has a cooperative DNA binding activity. Plant Cell 9(5):799-807

Suzuki M, Ketterling MG, Li QB, McCarty DR (2003) Viviparous1 alters global gene expression patterns through regulation of abscisic acid signaling. Plant Physiol 132(3):1664-1677

Thomas C, Meyer D, Wolff M, Himber C, Alioua M, Steinmetz A (2003) Molecular characterization and spatial expression of the sunflower ABP1 gene. Plant Mol Biol 52(5):1025-1036

Tiedemann J, Neubohn B, Muntz K (2000) Different functions of vicilin and legumin are reflected in the histopattern of globulin mobilization during germination of vetch (Vicia sativa L.). Planta 211(1):1-12

Van der Maarel MJ, Van der Veen B, Uitdehaag JC, Leemhuis H, Dijkhuizen L (2002) Properties and applications of starchconverting enzymes of the alpha-amylase family. J Biotechnol 94(2):137-155

Wilson ID, Barker GLA, Beswick RW, Shepherd SK, Lu C, Coghill JA, Edwards D, Owen P, Lyons R, Parker JS, Lenton JR, Holdsworth MJ, Shewry PR, Edwards KJ (2004) A transcriptomics resource for wheat functional genomics. Plant Biotechnol J 2:495-506

Wurmbach E, Yuen T, Sealfon SC (2003) Focused microarray analysis. Methods 31(4):306-316 